\title{
Cross-sectional understanding/research across clinical disciplines and integration of basic and clinical research
}

\author{
Yoshiki Hirooka ${ }^{1}$
}

(c) The Japan Society of Ultrasonics in Medicine 2019

This issue is scheduled to reach the readers around the end of July. I feel like the Japanese summers are getting hotter and hotter in recent years. I think the summer heat will be reaching its peak around the time you receive this issue. I hope that all the members of The Japan Society of Ultrasonics in Medicine are taking care of their health by getting plenty of rest, eating well, and staying hydrated.

This issue contains three review articles and wonderful original articles in the areas of basic and clinical research.

I specialize in the diagnosis and treatment of cancers located in the biliary tract and pancreas as a gastroenterologist. Of these cancers, pancreatic cancer in particular is known to be highly malignant. The only way to achieve complete remission of pancreatic cancer is to remove the entire tumor by means of surgery. What is troublesome about the treatment of pancreatic cancer is that recurrence is often seen, even when the tumor is completely removed via surgery from a histopathological standpoint. As such, post-resection anticancer chemotherapy is regarded as standard therapy. Moreover, performing anticancer chemotherapy prior to surgery is becoming more and more common now, even in patients who are eligible for surgery. In patients with inoperable pancreatic cancer, continuation of anticancer chemotherapy or radiation therapy as long as possible is common. The general rule of anticancer chemotherapy use is to administer as much of the drug as possible to the patient while keeping adverse drug reactions at an acceptable level. The most serious adverse drug reaction is thought to be bone marrow suppression. The primary focus is to use as much of the anticancer agent as possible by successfully controlling bone marrow suppression.

Cancer therapeutics-related cardiac dysfunction (CTRCD) is described in the review by Tanaka in this issue.

Yoshiki Hirooka

hirooka@med.nagoya-u.ac.jp

1 Department of Liver, Biliary Tract and Pancreas Diseases, Fujita Health University, Toyoake, Aichi, Japan
Anticancer agent-induced cardiac dysfunction has been reported since the 1960s. In the review, it states, "Currently, LV (left ventricular) dysfunction caused by cancer chemotherapy is known as CTRCD, which has become a leading cause of morbidity and mortality for cancer survivors, with the mortality rate for patients with CTRCD reportedly being as high as $60 \%$ by 2 years after treatment." This means that we have to pay close attention to CTRCD not only during treatment but also after treatment. The review concludes by stating that it is useful to perform echocardiography (global longitudinal strain (GLS)-guided assessment) for early prediction of CTRCD in patients who are scheduled to undergo anticancer chemotherapy. How many departments that perform anticancer chemotherapy, including gastroenterology, or how many doctors in those departments, are aware of this fact? Of course, I think it is true that some anticancer agents are more likely to cause CTRCD than others. Nevertheless, the reality seems to be that few doctors/institutions routinely perform echocardiography before anticancer chemotherapy. I think that being aware that CTRCD may actually be the cause of various adverse events that occur during anticancer chemotherapy may lead to a better understanding of this phenomenon across disciplines. I think that it may also lead to the conduct of research to verify this. Development of anticancer agents is advancing at a rapid pace. Against this backdrop, I think there are uncertainties over whether or not patients are prone to develop CTRCD. I think doctors who perform anticancer chemotherapy should always keep CTRCD in mind.

Articles from a variety of areas of clinical medicine are published in the journal of medical ultrasonics (JMU). Like the above review, reading articles about subjects outside one's specialty will broadens one's perspective, and may very well lead to new research that yields new findings that will help patients suffering from illness. In addition to clinical medicine, articles in areas of basic engineering related to ultrasound are also published in JMU. It is often the case that a seed in basic engineering bears fruit in clinical medicine 
to fulfill a patient need. It is not easy for one researcher to expose oneself to multiple areas of clinical medicine or basic engineering. We are proud to provide our readers with a wide scope and vast amount of information in JMU. We will continue to provide substantive articles going forward. I ardently hope that our readers will integrate and sublimate the information.
Publisher's Note Springer Nature remains neutral with regard to jurisdictional claims in published maps and institutional affiliations. 